\title{
REVISITING THE BOURGAIN-TZAFRIRI RESTRICTED INVERTIBILITY THEOREM
}

\author{
Peter G. Casazza And Janet C. TRemain
}

Abstract. We will give some new techniques for working with problems surrounding the BourgainTzafriri Restricted Invertibility Theorem. First we show that the parameters which work in the theorem for all $\|T\| \leqslant 2 \sqrt{2}$ closely approximate the parameters which work for all operators. This yields a generalization of the theorem which simultaneously does restricted invertibility on a small partition of the vectors and yields a direct proof that the Bourgain-Tzafriri Conjecture is equivalent to the Feichtinger Conjecture. We also fill in two gaps in the theory involving the relationship between paving results for norm one operators with zero diagonal and restricted invertibility results.

Mathematics subject classification (2000): Primary: 46B03, 46B07, 47A05.

Keywords and phrases: Restricted invertibility theorem, Bourgain-Tzafriri conjecture, Feichtinger conjecture, Kadison-Singer problem.

\section{REFERENCES}

[1] J. ANDERSON, Restrictions and representations of states on $C^{*}$-algebras, Trans. AMS, 249 (1979) 303-329.

[2] K. Berman, H. Halpern, V. Kaftal and G. Weiss, Matrix norm inequalities and the relative Dixmier property, Integ. Eqns. and Operator Theory, 11 (1988), 28-48.

[3] J. BOURGAIn AND L. TZAFRIRI, Invertibility of "large" submatrices and applications to the geometry of Banach spaces and Harmonic Analysis, Israel J. Math., 57 (1987), 137-224.

[4] J. Bourgain And L. TZAFriri, On a problem of Kadison and Singer, J. Reine. Angew. Math., 420 (1991), 1-43.

[5] P.G. CASAZZA, The art of frame theory, Taiwanese Jour. of Math., 42 (2000), 1-127.

[6] P.G. CASAZZa, O. Christensen, A. Lindner And R. Vershynin, Frames and the Feichtinger conjecture, Proceedings of AMS, 1334 (2005), 1025-1033.

[7] P.G. Casazza, M. Fickus, J.C. Tremain, And E. Weber, The Kadison-Singer Problem in Mathematics and Engineering: Part II: A detailed account, Operator Theory, Operator Algebras and Applications, Proceedings of the 25th GPOTS Symposium (2005), D. Han, P.E.T. Jorgensen and D.R. Larson Eds., Contemporary Math, 414 (2006), 299-356.

[8] P.G. CasazZa And J.C. TRemain, The Kadison-Singer problem in Mathematics and Engineering, PNAS, 1037 (2006), 2032-2039.

[9] P.G. CASAZZA AND R. VERShynin, Kadison-Singer meets Bourgain-Tzafriri, unpublished.

[10] O. Christensen, An introduction to frames and Riesz bases, Birkhauser, Boston, 2003.

[11] R. KAdison ANd I. Singer, Extensions of pure states, American Jour. Math., 81 (1959), 383-400.

[12] R. VERSHYNIN, John's decompositions: selecting a large part, Israel Jour. Math., 122 (2001), $253-$ 277.

[13] R. VERSHYNIN, Random sets of isomorphism of linear operators, IMS Lecture Notes - Monograph Series, High Dimensional Probability, 51 (2006), 148-154.

[14] N. Weaver, The Kadison-Singer Problem in discrepancy theory, Discrete Math., 278 (2004), 227 239. 